ÓSCAR ARCOS PALMA ÁLVARO ANDRÉS VERNAZZA PÁEZ editores
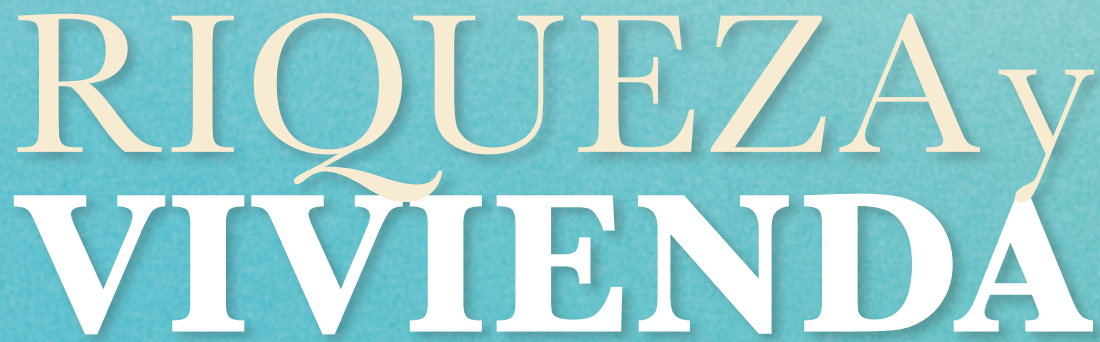

DESDE LA ECONOMÍA PÚBLICA, SOCIAL, FINANCIERA Y DE LAS ORGANIZACIONES
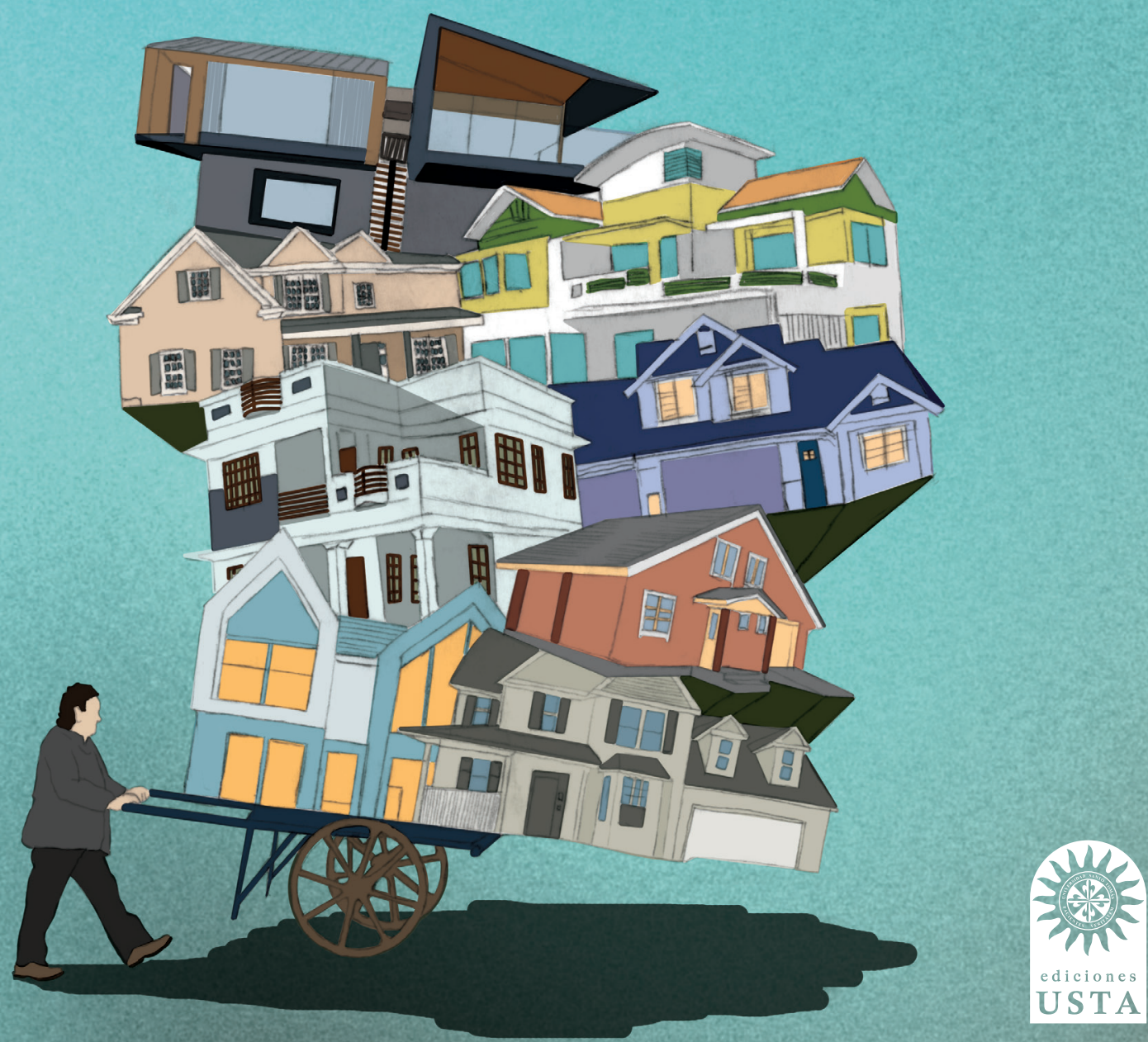




\section{Riqueza y vivienda Desde la economía pública, social, financiera y de las organizaciones}





\section{Riqueza y vivienda Desde la economía pública, social, financiera y de las organizaciones

\author{
Óscar Arcos Palma \\ Álvaro Andrés Vernazza Páez \\ Editores
}

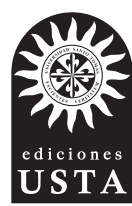


Arcos Palma, Óscar

Riqueza y vivienda: Desde la economía pública, social, financiera y de las

organizaciones/ Álvaro Andrés Vernazza Páez, Óscar Arcos Palma - Editores. Bogotá:

Universidad Santo Tomás, 2017.

162 páginas.

Incluye referencias bibliográficas

ISBN 978-958-782-053-9

E-ISBN: 978-958-782-054-6

1. Economía financiera 2. Economía clásica 3 Economía - Riqueza 4. Vivienda -- Aspectos sociales 5. Pobreza -- Necesidades básicas. 6. Epistemología.

Universidad Santo Tomás (Colombia).

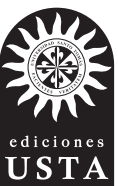

(C) Fray Juan Ubaldo López Salamanca O. P., Gustavo Adolfo Díaz Valencia, Álvaro Andrés Vernazza, John Álvaro Pérez Cruz, Esteban Pérez Caldentey, Óscar Arcos Palma, Normand Asuad, Mauricio Díaz Lozano, Lina María Penagos, Angélica Montes, Ruth Alejandra Patiño Jacinto, Olga Marina García Norato, Ana Hamón Naranjo, María Isabel Novo-Corti. Editores: Álvaro Andrés Vernazza Páez, Óscar Arcos Palma.

(C) Universidad Santo Tomás

Ediciones USTA

Carrera 9 n. ${ }^{\circ} 51-11$

Edificio Luis J. Torres, sótano 1

Bogotá, D. C., Colombia

Teléfonos: (+571) 5878797, ext. 2991

editorial@usantotomas.edu.co

http://ediciones.usta.edu.co

Coordinación de libros: Karen Grisales Velosa

Asistencia editorial: Andrés Felipe Andrade

Diagramación: Emilio Simmonds

Diseño de cubierta: Kilka Diseño Gráfico

Corrección de estilo: Henry Colmenares Melgarejo

Hecho el depósito que establece la ley

ISBN: 978-958-782-053-9

e-ISBN: 978-958-782-054-6

Impreso en Colombia $\bullet$ Printed in Colombia

Impreso por: Digiprint Editores S.A.S.

Primera edición: 2017

Todos los derechos reservados.

Se prohíbe la reproducción total o parcial de esta obra,

por cualquier medio, sin la autorización previa por escrito de los titulares. 


\section{Contenido}

$\begin{array}{ll}\text { Presentación } & 11\end{array}$

$\begin{array}{ll}\text { Prólogo } & 13\end{array}$

$\begin{array}{ll}\text { INTRODUCCIÓN } & 15\end{array}$

LA VIVIENDA COMO RIQUEZA INMOBILIARIA DE LOS HOGARES: ASPECTOS TEÓRICOS CONCEPTUALES Y CONTEXTUALES

Gustavo Adolfo Díaz Valencia

Álvaro Andrés Vernazza

John Álvaro Pérez Cruz

Esteban Pérez Caldentey $\quad 19$

Introducción $\quad 19$

Aspectos teóricos y conceptuales de la riqueza desde el punto de vista de la economía clásica 20

Diferencias entre riqueza e ingresos 23

La riqueza en vivienda y su relación con la política monetaria $\quad 26$

Mecanismo de transmisión de la política monetaria según Tobin 28

Relación de las crisis financieras y la política monetaria 30

Desigualdad en la distribución de la riqueza 32

Ciclos inmobiliarios $\quad 34$

Racionalidad y burbujas especulativas $\quad 38$ 
Aspectos metodológicos del análisis de

la presencia de burbujas inmobiliarias

Conclusiones

Referencias

ELEMENTOS TEÓRICOS SOBRE RIQUEZA Y VIVIENDA DESDE

LA PERSPECTIVA DE LA ECONOMÍA PÚBLICA

Óscar Arcos Palma

Normand Asuad

Introducción $\quad 49$

Sobre las nociones de ingreso, capital y riqueza 51

Hábitat, economía y política pública 52

Sobre la vivienda y el gasto social como compensación del ingreso individual

Contexto general de la política pública de vivienda

en Colombia

$\begin{array}{ll}\text { Conclusiones } & 64\end{array}$

$\begin{array}{ll}\text { Referencias } & 64\end{array}$

RIQUEZA Y VIVIENDA: ELEMENTOS TEÓRICOS DESDE

LA ECONOMÍA DE LAS ORGANIZACIONES

Mauricio Díaz Lozano

Lina María Penagos

Angélica Montes

Ruth Alejandra Patiño Jacinto $\quad 67$

$\begin{array}{ll}\text { Introducción } & 67\end{array}$

Una aproximación al concepto de riqueza desde la $\begin{array}{ll}\text { teoría institucional } & 70\end{array}$

Una aproximación al concepto de vivienda $\quad 82$

$\begin{array}{lr}\text { Conclusiones } & 89\end{array}$

$\begin{array}{ll}\text { Referencias } & 90\end{array}$ 
REFLEXIONES SOBRE POBREZA, RIQUEZA Y VIVIENDA, DESDE ALgunos PENSADORES GRIEgos, SANTO TOMÁS DE AQUiNo Y LOS CLÁSICOS

Olga Marina García Norato

Fray Juan Ubaldo López Salamanca O. P.

Ana Hamón Naranjo

María Isabel Novo-Corti 93

Introducción $\quad 93$

La noción de pobreza desde autores griegos, santo Tomás y los clásicos

La noción de riqueza $\quad 96$

$\begin{array}{ll}\text { La noción de vivienda } & 101\end{array}$

Interpretación contemporánea de las categorías:

pobreza, riqueza y vivienda en Colombia 104

$\begin{array}{ll}\text { Conclusiones } & 109\end{array}$

$\begin{array}{ll}\text { Referencias } & 112\end{array}$

APÉNDICE

FACUlTAD DE ECONOMÍA

Plan Estratégico de InVestigación 2OI7-2027 117

$\begin{array}{ll}\text { Introducción } & 117\end{array}$

1. Política de investigación de la Facultad de Economía 118

2. La investigación en el Plan Integral Multicampus

-PIM- 2016-2027 y en el Plan General de Desarrollo 2016-2019

3. Fundamentos epistemológicos de la investigación en la Facultad de Economía

4. Líneas activas y programas de investigación en la Facultad de Economía

5. Consolidación de líneas, programas

y grupos de investigación

Referencias 



\section{Presentación}

【 a Universidad Santo Tomás tiene el gusto de ofrecer este libro al —mundo académico y a la sociedad, producto de los avances de investigación que los profesores del grupo Economía y Humanismo de la Facultad de Economía han venido desarrollado durante los tres últimos años, en el marco del programa de investigación Distribución de la Riqueza y Equidad. Los problemas de la acumulación de la riqueza y de las desigualdades sociales han sido unas constantes a través de la historia de las sociedades, con características cambiantes y complejidades tan diversas que en nuestros días plantean preocupaciones y retos enormes a los gobiernos, a las organizaciones multilaterales, al ámbito académico y a los grupos sociales en el mundo.

El referente orientador más cercano en el tiempo para los trabajos que adelantan los investigadores de la Facultad de Economía es el pensamiento del padre Joseph Lebret O. P., expresado con meridiana claridad en sus reflexiones sobre economía y humanismo. El padre Lebret, en su peregrinaje por diversos países del mundo, meditó, investigó y ofreció como resultado de sus meditaciones e investigaciones, alternativas para el desarrollo de las sociedades, colocando al ser humano en el centro de toda acción humana. Los problemas de la acumulación irracional y desigual de la riqueza y las consecuencias que se manifiestan en desigualdades, exclusión y pobreza, estuvieron presentes en el pensamiento del padre Lebret. Los problemas que viven las sociedades 
denominadas del tercer mundo siguen siendo hoy los mismos a los que se refería el padre Lebret hacia los años cincuenta del siglo xx:

- Concentración de la tierra rural y la pobreza en el campo.

- Utilización irracional del suelo rural que provoca erosión y desgaste de la tierra.

- Limitaciones y posibilidades de la participación política.

- Fragmentación institucional y territorial de los Estados, proclive a la corrupción.

- Escaso interés en la investigación científica.

- Exclusión de la mayor parte de la población del acceso a la educación.

La noción de desarrollo basada en las capacidades de la gente está explícita en el pensamiento del padre Lebret: aboga por las condiciones del "ser", como categoría humana, y del "hacer", como expresión de las realizaciones individuales y colectivas y también como expresión de las libertades.

Confiamos en que los productos de la investigación que se encuentra en curso y cuyos primeros resultados se presentan en este libro, sean un aporte sustantivo para los hacedores de política en una época en que las sociedades enfrentan grandes retos de transformación social, cultural, política y económica. Y, de manera especial, para la situación de nuestro país, que nos demanda grandes compromisos en el largo período del posconflicto y la construcción de una sociedad reconciliada y en paz.

Fray Juan Ubaldo López Salamanca O. P. Rector General Universidad Santo Tomás 


\section{Prólogo}

Ta investigación en la Universidad Santo Tomás es un ejercicio per- 1 manente del conocimiento, abierto al cuestionamiento, al debate y a la búsqueda de soluciones de los problemas que afectan a la sociedad, para lo cual se inspira en el pensamiento de Tomás de Aquino, expresado en el lema institucional: facientes veritatem.

La investigación está orientada por la legislación nacional y en un marco epistemológico y normativo propio que se desarrolla en el Estatuto Orgánico, el Proyecto Educativo Institucional, la Política Curricular, el Estatuto Docente, el Proyecto Investigativo Institucional (PROIN) y en otros documentos de la legislación interna, y se constituye en una de las partes integrales de los Planes de Desarrollo Institucional.

La Facultad de Economía y la Maestría en Ciencias Económicas, se identifican con la orientación nacional, institucional y del Centro de Investigaciones Económicas Louis Joseph Lebret O. P. de la Facultad de Economía. En ese contexto se formuló a finales del año 2014 el Plan de Investigación 2015-2020, denominado: Distribución de la Riqueza y Equidad, con el propósito de diseñar y construir programas y proyectos de investigación orientados a generar nuevo conocimiento de aporte a la academia y para la solución de los problemas sociales, económicos, políticos e institucionales en materia de distribución de la riqueza, equidad y justicia social. El plan se guía por las cuatro líneas de investigación establecidas en la Facultad de Economía y lideradas 
por el grupo de investigación Economía y Humanismo: Economía Pública, Economía Social, Economía Financiera y Economía de las Organizaciones.

En particular, el proyecto (al que corresponden cada uno de los apartados que se presentan en este libro), se refiere al Análisis de la riqueza y su relación con la vivienda, y de manera más puntual, a los elementos teóricos que desde los enfoques de las cuatro líneas de investigación relacionan las categorías generales de riqueza y vivienda. El propósito de relacionar riqueza y vivienda conduce a indagaciones en la forma como la sociedad genera la riqueza a partir de sus posibilidades y de la manera como la sociedad misma está organizada y distribuye sus beneficios en la población. Remite de igual modo a la reflexión acerca de la pobreza y la exclusión social.

La ventaja de los avances de esta investigación es la fundamentación en economía y humanismo que los investigadores le han dado a este trabajo, bajo las ideas germinales que se encuentran en el pensamiento del padre Louis Joseph Lebret O. P., de la comunidad dominicana, el cual coloca en el centro de los debates la condición del ser humano. Los textos que contiene este libro se han elaborado bajo el pensamiento iluminador en economía y humanismo del padre Lebret. Estamos seguros de que sus aportes han de contribuir al debate académico y constituirán motivo de reflexión para las soluciones que nuestras sociedades requieren hoy en día en materia de convivencia, distribución de la riqueza, bienestar y justicia social.

Dr. Jorge Martínez Carvajal Decano Facultad de Economía

Dr. Álvaro Andrés Vernazza Páez Director Maestría en Ciencias Económicas 


\section{Introducción}

F ste libro constituye un primer avance de la investigación: AnáliUsis de la vivienda y su relación con la distribución de la riqueza en América Latina 1990-2014. Estudios de caso: Colombia, México, Chile y Bolivia, la cual se inscribe en el Plan de Investigación 20152020, Distribución de la Riqueza y Equidad, del grupo Economía y Humanismo de la Facultad de Economía, cuyo contenido se presenta en el apéndice de este libro. La investigación tiene por objeto analizar las relaciones dinámicas entre las firmas, los consumidores y el Estado en el mercado de la vivienda, estableciendo los alcances de la eficiencia económica, la acumulación de la riqueza y la conveniencia por el bienestar social. Se aborda desde cuatro enfoques analíticos que corresponden a las cuatro líneas de investigación de la Facultad de Economía: Economía Pública, Economía Social, Economía Financiera y Economía de las Organizaciones. A su vez, en cada uno de los enfoques se abordan en alguna medida las dimensiones macro, micro, meta y meso. En particular, tanto los enfoques como las dimensiones, la investigación se ha propuesto desarrollar los siguientes temas:

- Política de vivienda y riqueza (enfoque de economía pública y dimensiones macro y meso).

- Vivienda, pobreza y distribución del ingreso (enfoque de economía social y dimensiones micro y macro). 
- Agentes sectoriales, producción de vivienda y acumulación de riqueza (enfoque de economía de las organizaciones y dimensiones macro y meta).

- Burbuja financiera e inmobiliaria (enfoque de economía financiera y dimensión micro).

En el tema de la riqueza y la vivienda se observan relaciones claras desde las perspectivas macro, micro, meso y meta, las cuales permiten una comprensión global y holística de su problemática y de las soluciones que demanda. Las relaciones de los agentes en esas perspectivas son muy complejas. Desde el punto de vista micro, se enfatizan aspectos relacionados con la maximización de las rentas por parte de las firmas y la maximización de la utilidad por parte de los individuos.

En la perspectiva macro, se contrastan los aspectos micro en un contexto de dinámicas macroeconómicas relacionadas con las políticas monetaria y fiscal, esencialmente, en virtud de las relaciones causa-efecto que se presentan entre la actividad económica sectorial de la vivienda y los mercados inmobiliarios, con la tasa de interés, la inflación, la balanza de pagos, el déficit fiscal y el desempleo, entre otros aspectos de importancia. Desde la dimensión macro, los gobiernos regulan la actividad económica general. Tiene efectos directos en el comportamiento del empleo, los ingresos y el ahorro.

La perspectiva meso significa que a los componentes y relaciones micro/macro se añaden la actuación de los agentes, las reglas de juego, la articulación de intereses comunes, las alianzas, las estrategias y las acciones conjuntas que hacen posible su actuación en el mercado y las condiciones de infraestructura que facilitan la producción y el intercambio. El Estado es un agente más en el entramado de relaciones, con un rol particular definido en el modelo de desarrollo: la regulación de las actividades económicas. Los dos componentes que se conjugan entre lo micro y lo macro de una parte, y lo institucional normativo, de otra, son el máximo de la eficiencia económica -productividad y competitividad- y la conveniencia por el bienestar. Las iniciativas de política relacionadas con la dinámica sectorial de la vivienda y los programas de acceso a la misma orientados a los ciudadanos, tienen esos ingredientes. 
La eficiencia de las acciones realizadas en el nivel meso dependen del nivel meta: se define como la capacidad de concertación estratégica de los actores (cooperación público-privada entre autoridades públicas, comunidad organizada, empresarios) y la disposición de la sociedad a asumir desafíos relacionados con el desarrollo. El papel del Estado consiste en convocar y animar a los demás agentes a participar en los procesos económicos y sociales en beneficio de su desarrollo.

En ese horizonte analítico, el grupo de investigación Economía y Humanismo ha desarrollado los primeros avances de la investigación, referidos a la indagación teórica sobre vivienda y riqueza desde los cuatro enfoques a los que nos hemos referido, cuyos resultados se presentan en este libro. Así, el contenido del libro se estructura en los siguientes apartados:

- La vivienda como riqueza inmobiliaria de los hogares: aspectos teóricos conceptuales y contextuales.

- Elementos teóricos sobre riqueza y vivienda desde la perspectiva de la economía pública.

- Riqueza y vivienda: elementos teóricos desde el enfoque de la economía de las organizaciones.

- Reflexiones sobre pobreza, riqueza y vivienda, desde algunos pensadores griegos, santo Tomás de Aquino y los clásicos. 



\title{
La vivienda como riqueza inmobiliaria de los hogares: aspectos teóricos conceptuales y contextuales
}

\author{
Gustavo Adolfo Díaz Valencia* \\ ÁlVAro ANDrés VernaZZA** \\ John Álvaro Pérez Cruz*** \\ Esteban Pérez CaldenteY $\% * *$
}

\section{Introducción}

Fipe cátulo presenta aspectos generales del marco teórico que - sustenta el concepto de vivienda y su relación con la riqueza, explica el desarrollo de movimientos especulativos y su incidencia en el comportamiento de los precios, así como también la generación de ciclos que podrían generar burbujas inmobiliarias. Para tal fin, se analizan brevemente aquellos autores que desde la perspectiva clásica,

* Economista, Ph. D. en Ciencias Económicas, Universidad Nacional de Colombia. Docente investigador Maestría en Ciencias Económicas Universidad Santo Tomás.

** Economista, Ph. D. (c), Universidad de la Coruña, España. Máster Oficial en Banca y Finanzas, Universidad de la Coruña, España. Magíster en Ciencias Económicas, Universidad Santo Tomás. Director de la Maestría en Ciencias Económicas de la Universidad Santo Tomás.

*** Economista de la Universidad Católica. Máster en Administración y Finanzas Corporativas, Universidad de Viña del Mar, Chile. Docente Facultad de Economía Universidad Santo Tomás.

****Economista, Ph. D. en Economía. Jefe de la Unidad de Financiamiento de la Comisión Económica para América Latina-CEPAL. 
neoclásica y marginalista (como también algunos representantes de la escuela austriaca), han contribuido directa o indirectamente a la elaboración del concepto de riqueza inmobiliaria, la cual hace parte del patrimonio de los hogares, pero que en algunos casos su adquisición se convierte en un factor de exclusión.

Hablar de la riqueza inmobiliaria sugiere indagar sobre el manejo de la política monetaria, pues mediante la intervención en la tasa de interés se generan efectos sobre el gasto y el ingreso de los hogares, que de igual manera inciden el comportamiento del mercado de vivienda, lo cual afectaría no solo el consumo sino también pondría en riesgo la tenencia del activo, considerando que algunos propietarios son usuarios de créditos con el sistema financiero.

El capítulo presenta los siguientes subtemas: aspectos teóricos y conceptuales de la riqueza desde el punto de vista de la economía clásica; diferencias entre riqueza e ingresos; la riqueza en vivienda y su relación con la política monetaria; desigualdad en la distribución de la riqueza; ciclos inmobiliarios; y racionalidad y burbujas especulativas.

\section{Aspectos teóricos y conceptuales de la riqueza desde el punto de vista de la economía clásica}

El concepto de riqueza ha evolucionado a medida que las sociedades se transforman. Uno de los primeros autores en efectuar un acercamiento a este concepto fue Adam Smith. En su obra La riqueza de las naciones, el concepto de riqueza aparece como uno de los objetivos que debe alcanzar cualquier nación. Pero, ¿de dónde proviene la riqueza y cuáles son las formas de alcanzarla? Este es el interrogante que siempre se ha tratado de responder desde el desarrollo del comercio exterior y la división y la especialización internacional del trabajo. Por tal razón, Smith concluye que el trabajo es uno de los medios para alcanzar la riqueza, pues gracias a esta actividad el individuo puede obtener sus bienes y generar procesos de acumulación, entre más productivo sea el trabajo y más división haya, la riqueza será más cercana, es decir, existe una asociación entre riqueza y valor. 
La riqueza se mide en términos del trabajo que una persona puede disponer a través de sus bienes y, tal como lo afirmaba Adam Smith, un hombre "será rico o pobre de acuerdo con la cantidad de trabajo ajeno de que pueda disponer o se halle en condiciones de adquirir" (Smith, Libro I cap. V, p. 31). La obtención de la riqueza por las naciones provee la condición dinámica para la felicidad del individuo.

Por lo tanto, la riqueza se mide en términos de la cantidad de trabajo que una persona puede disponer para obtener los bienes necesarios para su bienestar. Smith manifiesta que: "Todo hombre es rico o pobre según el grado en que puede gozar por sí de las cosas necesarias, útiles y deleitables para la vida humana; y una vez introducida en el mundo la división del trabajo es muy pequeña parte la que de ellas puede obtener con solo el trabajo propio" (Smith, 1776, p. 49). La riqueza de las naciones no solo se basa en un aspecto material o monetario a partir de la división del trabajo, sino también en la búsqueda de felicidad del individuo, que otorga un grado de independencia en su forma de vivir, para alcanzar el bienestar. Cada individuo debe lograr este bienestar y de esta manera se obtiene el bienestar social.

David Ricardo, otro autor relevante de la teoría clásica, difiere de este concepto de Smith, al considerar que la riqueza y el valor como categorías diferentes de acumulación dependen del grado de dificultad que tenga el desarrollo de la actividad productiva (Ricardo, 1993, p. 205). La riqueza no puede ser estimada por la cantidad de trabajo que puede comprar. Al aumentar continuamente la facilidad de producción, disminuye constantemente el valor de algunas mercancías, pero con los mismos medios se adiciona riqueza nacional y aumenta el valor de la producción potencial. Ricardo advierte que en economía política han ocurrido equivocaciones al considerar que un aumento de la riqueza es lo mismo que un aumento del valor.

Ricardo considera que: "La riqueza de una nación puede ser incrementada de dos maneras: empleando una proporción mayor del ingreso en mantener el trabajo productivo, lo cual permitirá aumentar no solo la cantidad de valor y el volumen de mercancías y por ende sus riquezas" y, por otro lado, "incrementar la cantidad de trabajo productivo empleado, aumentará la riqueza pero no el valor” (Ricardo, 1993, 
pp. 205-210); en este sentido, un aumento del capital como parte de la riqueza para generar producción futura aumenta la riqueza. En efecto, la riqueza depende de la cantidad de bienes producidos sin tomar en cuenta la facilidad con que se hayan obtenido estos.

Karl Marx plantea una crítica a David Ricardo y a Adam Smith, en el sentido de que la fuerza de trabajo permite obtener riqueza a los capitalistas, a través de la plusvalía necesaria para la producción de mercancías y que no solo genera un aumento de su valor sino que también aumenta la riqueza sin participar en su distribución, lo que conlleva a una mayor desigualdad entre el asalariado y la tasa de ganancia que reciben los capitalistas. En su obra denominada El capital, comentaba que:

Dados la cuota de plusvalía o grado de explotación de la fuerza de trabajo y el valor de esta o la magnitud del tiempo de trabajo necesario, es evidente que cuanto mayor sea el capital variable tanto mayor será también la masa del valor y la plusvalía producidos. Dado el límite de la jornada de trabajo y dado también el límite del tiempo de trabajo necesario, la masa de valor y plusvalía que puede producir un capitalista determinado depende exclusivamente, como es natural, de la masa de trabajo que ponga en acción. Y esta, a su vez, depende siempre bajo los supuestos de que partimos, de la masa de fuerza de trabajo o del número de obreros que explote, el cual está, por su parte, condicionado por la magnitud del capital variable que este patrono desembolse. Dada la cuota de plusvalía y dado también el valor de la fuerza de trabajo, las masas de plusvalía producida se hallan, pues, en razón directa a las magnitudes del capital variable desembolsado (Marx, 2008).

La plusvalía genera un mayor valor al producto y contribuye al incremento de la tasa de ganancia del capitalista y por ende a su riqueza.

Franco Modigliani (1986), en su teoría del ciclo vital, ahorro individual y riqueza de las naciones, establece que la riqueza se alimenta del ingreso, el ahorro y las herencias. Durante el ciclo de vida laboral de una persona la cotización en pensiones es otra fuente de ahorro 
que le permite disfrutarlo al momento de la jubilación. Lo que resalta este autor es que la riqueza es el resultado de diferentes decisiones de ahorro provenientes de la vida laboral, el cual es invertido en diferentes activos financieros y reales a través de los fondos de pensiones e inversiones de cartera, permitiendo incrementar la rentabilidad y ampliar la capacidad de reserva de los agentes, mejorando su bienestar y riqueza.

Cuando una economía está en continuo crecimiento, que en equilibrio es igual a la tasa de crecimiento de la riqueza, la tasa de ahorro es proporcional al crecimiento (ver ecuación 1) y la población joven es esencial en el proceso de generación del ahorro; si mantienen una tasa constante de consumo, este ahorro es fuente generadora de inversión y puede facilitar la adquisición de la vivienda de manera más fácil, mientras las personas jubiladas efectúan el consumo a partir de su pensión.

Según Modigliani, "el comportamiento de las tasas de ahorro se puede deducir de la de riqueza privada total, $W$, a través de la relación $S=A W$, lo que implica:

$$
s=\frac{S}{Y}=\frac{\Delta W}{W} \frac{W}{Y}=\rho w, \frac{d s}{d \rho}=w+\rho \frac{d w}{d \rho}
$$

Donde $w$ es la relación de la riqueza con respecto al ingreso, $s$ es la tasa de ahorro y $\rho$ es la tasa de crecimiento de la economía que en el estado de equilibrio es igual a la tasa de crecimiento de la riqueza $\frac{\Delta W}{W}$. Puesto que $w$ es positivo y se basa en un consumo de ciclo de vida y el nivel de ingresos, el crecimiento de la riqueza depende del nivel de ingresos" (Modigliani, 1986).

\section{Diferencias entre riqueza e ingresos}

La riqueza se puede definir como un stock dado en un momento de tiempo, el cual puede ser material y laboral, donde el individuo puede obtener los bienes materiales que en cierta medida garantizan su bienestar. En cambio, el ingreso es un flujo de ganancias, el cual se obtiene de manera importante como compensación al trabajo, los rendimientos del capital, los alquileres o los intereses que se obtengan en 
el sistema financiero. Sin embargo, la riqueza puede tomar diferentes formas de acuerdo con las posibilidades naturales o artificiales existentes en el tiempo.

Se pueden clasificar varios tipos de riqueza, como la humana formada por los salarios después de impuestos, y la riqueza no humana, compuesta por la riqueza inmobiliaria y la financiera; ambas conforman la riqueza total. Esta clasificación se ha tomado en cuenta para analizar su efecto en la función consumo.

Una de las clasificaciones que permite llegar a cuantificar el concepto de riqueza y vincularlo con el ingreso, la plantea Piketty en su obra El capital en el siglo XXI (2013), en la cual estudia la riqueza como el valor del capital nacional y lo cuantifica en términos del ingreso nacional.

Piketty toma como referencia algunos postulados propuestos por Marx en El capital, para establecer una relación entre el valor del capital nacional con la riqueza y el ingreso. Por tal razón, afirma que el capital está relacionado con la riqueza y por lo tanto, la riqueza nacional se puede desagregar como la suma del capital nacional más el capital extranjero neto. De tal manera que, el capital nacional se define como el valor del stock del capital ubicado dentro de las fronteras de un país el cual incluye viviendas y tierras.

"Capital nacional = tierras agrícolas + viviendas + otro capital interno + capital extranjero neto" (Piketty, 2014).

Con la anterior clasificación, analiza el comportamiento del capital nacional a lo largo del siglo xx para Francia, Inglaterra y Estados Unidos, llegando a la conclusión de que los porcentajes de participación de los anteriores factores dentro del valor del capital nacional, cambian de forma dramática durante el último siglo, gracias a la transformación de economías agrícolas a economías industriales, lo cual elimina el peso de la tierra agrícola y se observa el desplazamiento hacia otro tipo de bienes, en especial la vivienda, otro capital interno y capital extranjero. 
Otro de los autores que analiza el concepto de la riqueza inmobiliaria y el ciclo laboral es Franco Modigliani, quien, en la teoría del ciclo vital, recalca el papel de la inversión en activos financieros o inmobiliarios de largo plazo en la generación de riqueza y su relación con la inversión en fondos financieros o finca raíz. Este concepto de riqueza asociado a la tenencia de vivienda está relacionado estrechamente a la teoría de la renta permanente y el ciclo vital, donde el ahorro es la forma de cubrir las variaciones intertemporales del consumo. Entonces, las familias ahorran a partir de su ingreso disponible y los intermediarios financieros canalizan este ahorro básicamente para la inversión, que en muchos casos se hace para compra de vivienda.

En algunas ocasiones la compra de vivienda requiere un financiamiento propio a través del ahorro o el endeudamiento, razón por la cual la demanda del sector inmobiliario está estrechamente vinculada a las necesidades de financiamiento que ofrece el sistema financiero, dado que el precio de la vivienda es superior al ingreso disponible de muchos hogares.

El estudio de los ciclos inmobiliarios y sus efectos macroeconómicos ha permitido destacar el efecto riqueza. En ese horizonte, existe interés en estudiar el efecto riqueza de la economía a partir del aumento de la riqueza de las familias asociado a la revalorización de la vivienda equivalente a la expansión del ciclo inmobiliario.

En los últimos años las decisiones de ahorro y consumo de los hogares se han visto condicionadas por la incertidumbre del sistema económico, donde se ha presentado una tendencia al incremento del stock de activos financieros a pesar de ahorrar una menor proporción del ingreso disponible por precaución, lo que ha conllevado a un aumento de la riqueza financiera aunque esta distribución no sea homogénea.

En Colombia ha sido vigoroso el crecimiento de la riqueza financiera dado el resultado directo de los cambios que ocurrieron con los procesos de globalización económica y financiera y que propiciaron la creación de los fondos privados de pensiones, generando un aumento en las pensiones y el ahorro de dinero. Así, las familias pueden mantener su riqueza de diversas formas, comprando acciones, efectuando 
depósitos bancarios, invirtiendo en certificados de depósito a término, adquiriendo fondos de inversión o en fondos de pensiones voluntarias, mientras otra parte está contenida en vivienda. Por tal razón, dentro del cálculo de la riqueza, se contabiliza, además de la vivienda, la riqueza financiera de los hogares. La vivienda se considera como un bien básico y tiene una estrecha relación con la tasa de interés, pues incide en la compra de finca raíz y finalmente este activo es una fuente de riqueza.

\section{La riqueza en vivienda y su relación con la política monetaria}

La riqueza inmobiliaria se puede considerar como un canal de transmisión de la política monetaria, que induce a una modificación de la tasa de interés de intervención, genera una variación en el costo de uso de la vivienda y por ende, en el consumo de los hogares. Esta situación se conoce como el efecto riqueza, la cual aplica cuando los hogares acumulan y posteriormente agotan su riqueza con el fin de mantener el consumo.

Según Mishkin, el mercado de la vivienda es una preocupación central para las autoridades monetarias. Para lograr el objetivo de la estabilidad de precios, el banco central debe entender el papel que juega la vivienda como mecanismo de transmisión monetaria, donde la tasa de interés es el principal canal relacionado con la adquisición de vivienda, y es el mecanismo que influye directamente en el costo de uso de la misma, las expectativas de los movimientos de los precios de oferta y demanda para la adquisición de la vivienda futura e indirectamente influencia la economía real a través de efectos de riqueza. Por ello, los hacedores de la política deben considerar la interacción de la estabilidad financiera con el mecanismo de transmisión monetaria, y a su vez discutir las formas en que el sector de la vivienda puede ser una fuente de inestabilidad financiera, y si dicha inestabilidad podría afectar la capacidad de un banco central para estabilizar la macroeconomía global.

El manejo de la política a través de las tasas de interés a corto plazo, afecta directa o indirectamente no solo el comportamiento económico 
general sino también el mercado de la vivienda, a través de por lo menos seis canales (Mishkin, 2007):

1. Los efectos directos de las tasas de interés en el costo de uso del capital.

2. Las expectativas de los futuros movimientos de precios de las viviendas.

3. La oferta de vivienda.

4. Los efectos en la riqueza del hogar cuando sube el precio de la vivienda.

5. El efecto de los créditos sobre el gasto de los consumidores.

6. Los efectos del crédito en la demanda de vivienda.

El efecto directo de la tasa de interés a través del costo de uso del capital del inmueble es un determinante importante de la demanda y también afecta sensiblemente el pago de la obligación.

Cuando la política monetaria aumenta la tasa de interés a corto plazo, las tasas de interés a largo plazo también tienden a subir porque están vinculadas a la tasa de interés esperada de corto plazo; por consiguiente, si el costo de uso del capital aumenta y la demanda de vivienda cae y conduce a una disminución en la construcción de viviendas, la demanda agregada en la economía disminuye. Este canal de transmisión de la política monetaria es muy importante en modelos macroeconómicos utilizados por los bancos centrales, pero la gama de elasticidades estimadas de la inversión residencial al costo de uso del capital es amplia.

El costo de uso del capital $u c$ tiene en cuenta variables como: el precio de la vivienda $p h$, la tasa de la hipoteca $i$, el valor esperado de la apreciación del inmueble $\pi_{h}^{e}$ y las tasas de depreciación de la casa $\delta$ e interés después de impuestos $(I-t) i$, las cuales determinan el valor real del inmueble en ausencia de arbitraje y permiten establecer el rendimiento esperado, que para el propietario representa el alquiler del inmueble (Mishkin, 2007, p. 5):

$$
u c=p h\left[(I-t) i-\pi_{h}^{e}+\delta\right]
$$


El segundo término del costo de uso del capital, la tasa real de apreciación esperada del precio de la vivienda, $\pi_{h}^{e}-\pi^{e}$, describe otra manera como la política monetaria afecta a la actividad inmobiliaria (ver fórmula 2). Los cambios en estas expectativas pueden tener un efecto importante en el costo de uso de capital y por lo tanto en la demanda de vivienda, como Case y Shiller (2003) han puesto de relieve. Cuando la política monetaria se contrae y las tasas de interés suben, los precios de la vivienda se ablandan debido a que la demanda para la vivienda disminuye a través del mecanismo de transmisión del costo de utilización, como se ha descrito anteriormente.

Las expectativas de un futuro endurecimiento de la política monetaria, podrían bajar la esperada tasa real de apreciación del precio de la vivienda, aumentando así el costo de uso del capital actual, lo que daría lugar a una disminución de la demanda de vivienda y una disminución de la construcción de vivienda nueva (Mishkin, 2007, p. 5):

$$
u c=p h\left\{(I-t) i-\pi_{h}^{e}+\delta\right\}\left\{\pi_{h}^{e}-\pi^{e}\right\}
$$

En el sentido keynesiano, un incremento de la tasa de liquidez dada una política monetaria contractiva, estimularía la compra de bonos pero afectaría la demanda de inversión y el costo de uso del capital, lo cual induce a pensar que los hogares se abstendrán de comprar vivienda a menos que estén dispuestos a asumir el riesgo del crédito que genera el flujo de pago de la obligación.

También son considerados como parte de la riqueza de muchos hogares los bienes durables diferentes a la vivienda, los cuales son adquiridos con un propósito de consumo durable y no de valor, desde el punto de vista de la clasificación económica en que estos bienes son considerados.

\section{Mecanismo de transmisión de la política monetaria según Tobin}

Dentro del análisis de los movimientos de los precios de los activos, necesario para poder contextualizar los diferentes desencadenantes de las crisis financieras y con el fin de entender cómo el mercado se ve afectado por las decisiones de los hacedores de política, el estudio de 
los mecanismos de transmisión de la política monetaria permite entrever algunas posibles causas que pueden haber contribuido con la gestación de las burbujas al afectar el precio de los activos.

Tobin, en su artículo titulado: Monetary policies and the economy: the transmission mechanism (1978), estudia el efecto de la política monetaria sobre el valor de los activos, con el fin de establecer cómo la política monetaria puede afectar los precios en los mercados financieros.

Establece una relación entre el valor de mercado de un activo y su costo de reposición, a la cual denominó $q$. Este indicador es una medida que resume el impacto de los mercados financieros y la compra de bienes durables.

El índice $q$ al momento de su primer cálculo (cuando numerador y denominador son iguales) y en economías en equilibrio, debería tener un valor de 1 , no obstante el valor normal de $q$ es superior a 1 por la capitalización de las ganancias que se tienen del activo durante el tiempo, aumentando su valor de mercado.

La $q$ puede representar la eficiencia marginal del capital o el costo financiero de capital, es decir, la tasa a la cual los inversionistas descuentan los futuros rendimientos de una inversión. Si la $q$ es baja, puede desincentivar la inversión en esos activos, incluso para su reemplazo, dado que su valor de mercado estaría por debajo del de reposición.

En el mercado inmobiliario, Tobin asevera que este suceso también se cumple, ya que el valor de mercado de los inmuebles refleja el valor presente de las rentas actuales y futuras que estos pueden generar.

Asegura que la decisión de construcción de nuevas viviendas estará condicionada por el valor de las actuales (valor de mercado), ya que será rentable construir una nueva unidad, siempre que su costo de construcción (reposición) sea inferior al valor de mercado de la misma. En otras palabras, si el valor de construir una nueva unidad es mayor al de comprar una vivienda ya construida de similares características, no habrá incentivos para hacerlo, gracias a que el valor de reposición sería mayor al de mercado reduciendo así la $q$ inmobiliaria.

De otra parte, afirma que el factor que hace que los valores de los bienes (mercado y reposición) se ajusten en el tiempo, es la tasa de rentabilidad esperada por los inversionistas, y que dicha tasa depende de 
la valoración por parte de estos últimos, sobre la incertidumbre de los flujos futuros del activo y sus relaciones con la economía y el entorno, es decir, no es una tasa fijada por el Estado o el banco central.

Concluye que la tasa de rentabilidad esperada se ajusta al costo de capital, ya que involucra el costo de la deuda y del capital propio, por lo tanto difiere entre propietarios de los activos.

De acuerdo con lo descrito por Tobin, el banco central influencia dicha tasa de forma indirecta, ya que al modificar las tasas de instrumentos de corto plazo estas afectan por una parte el costo de la deuda y por otro lado generan un desplazamiento de la inversión hacia otros activos financieros, afectando así el costo de capital de los propietarios y por ende, afectando el valor de mercado de la vivienda, haciendo que el factor $q$ caiga.

De otro lado, las expectativas inflacionarias disminuyen la tasa de interés real, definida como la diferencia entre la tasa nominal y la inflación, haciendo que el costo de capital disminuya produciendo un incremento del valor del mercado y por lo tanto un crecimiento de $q$.

No obstante, dado que la política monetaria busca contrarrestar los efectos inflacionarios a través de una contracción monetaria, los efectos se verán en una disminución del valor de los activos, ya que el público preferirá sustituir estos bienes por activos de renta fija, gracias al incremento de la tasa generado en la política de contracción afectando así el valor de $q$. El costo de capital de los inversionistas se verá afectado por la contracción monetaria y el encarecimiento del costo de capital (Tobin, 1978).

\section{Relación de las crisis financieras y la política monetaria}

Un común denominador sobre las historias de las crisis financieras y la posibilidad de generación de burbujas en los precios de las viviendas, son los cambios de la política económica, en especial de la política monetaria, y la desregulación a nivel de los controles de divisas y la movilidad de los flujos de capital a las distintas economías. La razón puede 
estar relacionada con que el ingreso disponible puede ser aumentado o disminuido por medio de los instrumentos de la política monetaria.

Desde el punto de vista teórico, Von Mises, quien hace parte de la segunda generación de los gestores de la escuela austriaca, señala en su obra The theory of money and credit, que los bancos centrales en ocasiones crean dinero para incentivar los créditos a través de la tasa de interés. El efecto que puede traer el aumento de la oferta de dinero, en el mercado inmobiliario, es que las personas tengan facilidad para acceder al crédito y gracias a esa mayor liquidez proporcionada por la deuda, se presiona la demanda de bienes, generando inflación en los activos inmobiliarios. Los bancos han jugado un papel importante en la financiación de la actividad económica, si la tasa de interés de los prestamos está por debajo de la natural entonces incentiva el otorgamiento de los créditos y estos a su vez incentivan la demanda de nuevas inversiones incluyendo la vivienda, pero en el mediano plazo induce a un aumento de sus precios, lo cual podría desencadenar en una crisis si los agentes no cumplen con el pago de sus obligaciones.

Von Mises dirige una crítica a la escuela inglesa denominada “Currency School” la cual trató de explicar el auge del ciclo económico a través de "la emisión del dinero sin respaldo metálico" (Von Mises, 2005, p. 1). Además, advierte que esta escuela no tuvo en cuenta el papel de las cuentas corrientes en la emisión secundaria del dinero mediante el incremento de la apertura de las cuentas corrientes en época de expansión, por tal motivo para contrarrestar la crisis la legislación debía restringir la emisión sin respaldo metálico.

Von Mises trata de explicar el ciclo a partir del incremento de la emisión monetaria a través de la emisión de medios fiduciarios -no necesariamente respaldados por oro o depósitos en cuentas corrientes-, mecanismo que podría reducir la tasa de interés incluso por debajo de la interbancaria. El objetivo de esta medida respaldada en la emisión de títulos fiduciarios, es estimular la actividad económica en época de depresión, que podría impulsar el desarrollo de la actividad de la construcción, haciendo rentables los proyectos de inversión inmobiliaria. 
Sin embargo, la expansión del crédito no se puede presentar de manera indefinida, en tanto el desarrollo de las actividades productivas tiene un límite, dado que el incremento desbordado de los créditos puede generar simultáneamente un aumento de los precios. Igual sucede con la tasa de cambio, detonantes de una posible recesión de la actividad económica, dado que para muchas personas no resulta rentable tener dinero en efectivo, quienes motivados por los créditos fáciles pueden efectuar una serie de inversiones injustificadas y justificadas por la disminución artificial de la tasa de interés. Así, las crisis y la expansión de créditos que se reflejan en las bajas tasas de interés, generan inversiones innecesarias y posteriormente producen aumentos en los precios (Von Mises, 2005).

Hayek, también representante de la escuela austriaca, señala en su obra La teoría monetaria y el ciclo económico (1929), que el descenso del interés causado por una laxitud monetaria, promueve inversiones poco razonables en el auge, de tal forma que posterior a la disminución de las tasas de interés se pueden generar presiones no justificadas en los precios de los activos.

\section{Desigualdad en la distribución de la riqueza}

Piketty, en su libro El capital en el siglo XXI (2013), considera que la economía debe volcarse a abordar temas como la desigualdad y el empleo y por tal razón en uno de sus apartes estudia el problema de la distribución de la riqueza y de las rentas del capital, considerando estas más concentradas que la distribución de los ingresos del trabajo. Afirma que a largo plazo la clase media es el grupo que incide significativamente en la distribución de la riqueza, pues es un sector sensible a mejorar o empeorar su situación dependiendo de la estabilidad laboral que posea, por lo tanto, si se mantiene estable, su riqueza estará garantizada, la cual incluye la tenencia de vivienda adquirida a través de la financiación; pero si pierde su vinculación laboral su riqueza estará comprometida. 
Piketty considera que una de las soluciones al problema de la redistribución y la regulación de la riqueza mundial es la inmigración, especialmente en Estados Unidos y en algunos países de Europa, pues muchos inmigrantes provenientes de África y de determinadas naciones de Asia y América Latina, consideran que una mejora al acceso de estabilidad laboral y riqueza es instalarse en aquellos países que cuenten con mejores perspectivas de crecimiento; sin embargo, esta búsqueda de nuevas formas de vida se enfrenta a múltiples restricciones. Por lo tanto, la redistribución a partir de la inmigración no necesariamente resuelve el problema de distribución de la riqueza y mejora en las condiciones de vida incluyendo el acceso a la vivienda, y conlleva a que muchos de estos países propongan un nuevo tipo de regulación, sustentada en un Estado social con impuestos progresivos sobre la renta y el capital (Piketty, 2013).

Sobre la base anterior, la obra El capital en el siglo XXI busca también responder si la dinámica de la acumulación del capital privado conduce a un proceso de acumulación de riqueza más concentrada y a una mayor desigualdad y exclusión. Este aspecto se convierte en uno de los temas fundamentales de investigación de las ciencias económicas y en particular, de la economía social.

Piketty propone una relación entre el ahorro, el crecimiento y el ingreso nacional, a la cual llama "la segunda ley fundamental del capitalismo", donde establece que la relación entre capital/ingreso aumentará en el largo plazo gracias a la relación entre el ahorro $S$, dividido entre la tasa de crecimiento G. A diferencia de Marx, Piketty considera que la tasa de crecimiento no decae en el tiempo, con lo cual la acumulación puede llegar a generar una acumulación de capital tan alta que afectará de manera importante la desigualdad social.

Dentro de su análisis de la acumulación de capital de los países europeos, en especial de Inglaterra, propone que en el siglo xx, la acumulación de capital generada gracias a la colonización de los siglos pasados, permitió que los países conquistadores tuvieran un incremento de sus capitales gracias a que considera que el capital acumulado en el pasado, en el largo plazo, toma una importancia tal que aumenta las desigualdades de forma importante. 
Otras de las preocupaciones relacionadas con el tema de la desigualdad, son las dificultades que se presentan en la generación de riqueza y su relación con el bienestar de la población. Los procesos de acumulación son diferentes, en el sentido de que cada individuo busca apropiarse de la mayor cuota de bienes materiales y financieros para aumentar su bienestar, pero la suma de cada bienestar no contribuye al bienestar general tal y como lo afirmaba la escuela marginalista. Esta preocupación motiva a definir un análisis sobre las implicaciones que ocasiona el problema de la distribución de la riqueza a nivel mundial y su condicionamiento en el desarrollo económico, dejando rezagada la parte social.

Según Amartya Sen, las características de la desigualdad en distintos espacios, como ingresos, riquezas, felicidad, etc., tienden a distanciarse unas de otras dada la heterogeneidad de la gente. "La igualdad de oportunidades puede llevar a ingresos muy desiguales. Unos ingresos idénticos pueden ir acompañados de diferencias significativas de riqueza. La misma cantidad de riqueza puede coexistir con diferentes niveles de felicidad" (Senn, 2011). La diversidad humana está relacionada con conflictos individuales, para evaluar la igualdad, la eficiencia y la justicia. Juzgar la igualdad y la eficiencia desde la perspectiva de la realización, supone otro enfoque desde el punto de vista tanto del utilitarismo como del bienestar, entonces el concepto de riqueza se puede analizar desde las realizaciones. Sobre esta base teórica, la vivienda concebida como parte de la riqueza puede generar realizaciones y ser un indicativo de oportunidad e igualdad, aunque para su realización es necesario disponer de un ingreso, ya que la dificultad radica en su generación, que es desigual.

\section{Ciclos inmobiliarios}

La teoría del ciclo inmobiliario está relacionada con la teoría del ciclo económico, dado que las oscilaciones recurrentes de la actividad económica y las fases que la caracterizan que van de la expansión a la contracción resultante de crisis, afectan sustancialmente el comportamiento del sector de la construcción y por ende el mercado de vivienda. 
Estos ciclos pueden ser resultado de cambios en las actividades financieras, de inversión, comerciales y monetarios.

Se destacan dos tipos de ciclos: los reales (cambios en la inversión, la tecnología, las actividades comerciales) y los monetarios. Lo ciclos económicos reales generados por cambios en la tecnología y el gasto producen impactos que afectan sustancialmente la producción efectiva respecto a la potencial y separan transitoriamente su posición de equilibrio a largo plazo; para el desarrollo de este tema se destacan autores como Finn E. Kyland y Edward C. Prescott. Mientras, los ciclos financieros caracterizados por la inestabilidad en los mercados financieros y su interacción con la actividad económica, se basan en la teoría keynesiana de la inversión y el ciclo económico, centrando su análisis en el papel del crédito antes que en el dinero para financiar la inversión, siendo su representante más importante Hyman Minsky (1992).

La teoría del ciclo vital (The life cycle hypothesis of saving, the demand for wealth and the supply of capital) de Franco Modigliani (1966), es otra teoría asociada al comportamiento de los ciclos económicos y tiene alguna relación con el mercado inmobiliario, considerada como el sustrato dominante que explica lo que ocurre en una economía con productos financieros que se invierten a largo plazo como hipotecas, viviendas y fondos de pensiones, entre otros. Sobre esta base, la adquisición de la vivienda se convierte en el activo más importante que consolida la riqueza de las familias y sirve también como garantía para facilitar los procesos de financiación que ofrecen las entidades crediticias. Dado que el concepto de vivienda propuesto por Modigliani está asociado a la teoría del ciclo vital y la renta permanente, el ahorro es un mecanismo fundamental que permite suavizar la senda intertemporal del consumo. Entonces, las familias ahorran a partir de su ingreso disponible y los intermediarios financieros canalizan este ahorro básicamente para la inversión, que en muchos casos se hace para compra de vivienda.

La escuela austriaca también ha contribuido al desarrollo de la teoría de los ciclos, destacándose autores como Friedrich Hayek, quien considera que los factores monetarios son importantes para la explicación del ciclo económico. En primera instancia encuentra una relación 
cercana entre el desarrollo económico y los créditos bancarios como forma de lograr este objetivo, y considera que: "Mientras hagamos uso de crédito bancario como un medio para fomentar el desarrollo económico, se deberán soportar los ciclos comerciales resultantes" (Hayek, 2008, p. 6). También establece que: las tasas de interés, los beneficios y la liquidez de los bancos son variables que permiten profundizar en los factores que determinan la expansión del crédito, así como nuestro conocimiento de sus límites, y por lo tanto prever los movimientos de los factores que determinan el desarrollo total de la situación económica (Hayek, 2008). Bajo este contexto instauró la primera relación entre la teoría monetaria y el ciclo económico.

Para facilitar la explicación del concepto de ciclo inmobiliario, es importante establecer su relación con algunas teorías del ciclo económico y la formación de burbujas inmobiliarias en fases expansivas. La explicacion de los ciclos inmobiliarios, derivada de algunas teorías del ciclo económico, ha estado asociada al comportamiento de los precios de la vivienda, siendo este un indicador importante que explica el comportamiento de la estabilidad macroeconómica y financiera de un país y su relación con el sector de la construcción. A su vez, el comportamiento de los precios está relacionado con el otrogamiento de créditos hipotecarios y la compra de vivienda, la interrelación de estas variables de alguna manera explica el ciclo inmobiliario. Períodos de auge han estado asociados al boom de la construcción relacionado con masivos planes de financiación, generación de fuentes de empleo y estabilidad macroeconómica, que permite facilitar la compra de vivienda en todos los sectores.

Por lo anterior, generalmente la compra de vivienda requiere un financiamiento propio a través del ahorro o el endeudamiento con la banca, por lo tanto la demanda del sector inmobiliario está estrechamente vinculada a las necesidades de financiamiento que ofrece el sistema financiero, dado que el precio de la vivienda es superior al ingreso disponible de muchos hogares. Entonces, la demanda de vivienda tiene una estrecha relación con el precio y los ingresos disponibles de los hogares y las tasas de interés, de tal manera que el comportamiento 
del ciclo inmobiliario está estrechamente asociado al sector bancario y al comportamiento macroeconómico de la economía.

Autores como Moneo Abreu (2004), determinan cuatro fases del ciclo inmobiliario: fases de corrección y estabilización en la parte inferior del ciclo y la fase de desarrollo de proyectos y creación de sobreoferta en la parte superior del ciclo; de acuerdo al comportamiento del mercado inmobiliario se desarrolla cada una de estas fases. En la fase de correción hay sobreoferta en el mercado de alquiler y las condiciones macroeconómicas son deficientes, hay aumento del desempleo y aplazamiento en la realización de nuevos proyectos inmobiliarios; la banca restringe los créditos y los precios de las viviendas empiezan a descender. En la fase de estabilizacion del mercado, el sector de la construcción reacciona pero con retraso, y las condiciones económicas y financieras de recuperación se presentan de manera lenta (Moneo Abreu, 2004).

En la fase de expansión se presentan dos subfases, la primera es de promociones y proyectos inmobiliarios, asociada a una recuperacion económica y mayor demanda de espacios para construcción, sin embargo los retrasos en este proceso hace que la demanda no se satisfaga completamente, los bancos incentivan el uso de créditos para construcción y compra de vivienda nueva y usada y se impulsa el desarrollo de nuevos proyectos de inversión inmobiliaria. La última subfase que es de sobreferta, entra al mercado con muchas promociones de vivienda, para estimular la compra de esta, y asimismo los bancos propician condiciones para el desembolso de recursos que faciliten la financiación (Moneo Abreu, 2004).

Arriaga (2012), señala que los ciclos inmobiliarios se transmiten a la actividad económica por tres vías: 1) el efecto riqueza en el consumo de las familias, 2) el efecto renta dado que el auge de la construcción genera un efecto en otros sectores de la economía aumentando el empleo y el ingreso y, 3) el canal financiero, en el que la estabilidad del sector bancario se ve afectada por la fluctuación del ciclo inmobiliario, donde el riesgo crediticio aumenta en la epoca del boom, cuando está en la fase creciente, aumenta la entrega de créditos y los riesgos si las condiciones bajo las que se otorgan no son restrictivas. 
Es importante destacar la distribución de la riqueza inmobiliaria en el contexto del estudio de los ciclos inmobiliarios y sus efectos macroeconómicos sobre la riqueza. Es por ello el interés de estudiar el efecto riqueza de la economía a partir del aumento de la riqueza de las familias, que está asociado a la revalorización de la vivienda equivalente a la expansión del ciclo inmobiliario, lo cual se ha reflejado en un aumento de la demanda y por lo tanto, de los precios.

En los últimos años las decisiones de ahorro y consumo de los hogares se han visto condicionadas por la incertidumbre del sistema económico, donde se ha presentado una tendencia al incremento del stock de activos financieros a pesar de ahorrar una menor proporción del ingreso disponible por precaución, que ha conllevado a un aumento de la riqueza financiera aunque esta distribución no sea homogénea.

Las familias pueden mantener su riqueza de diversas formas, pueden comprar acciones, mantener depósitos bancarios, comprar fondos de inversión, mientras otra parte está contenida en vivienda. Por tal razón dentro del cálculo de la riqueza se contabiliza además de la vivienda la riqueza financiera de los hogares. La vivienda se considera como un bien básico y tiene una estrecha relación con la tasa de interés pues incide en la compra de finca raíz y finalmente este activo es una fuente de riqueza.

\section{Racionalidad y burbujas especulativas}

En la teoría económica marginalista, los agentes son considerados como racionales por cuanto buscan maximizar la utilidad de su consumo y en general su bienestar. Ahora, al considerar la vivienda como parte del patrimonio de las familias y de la riqueza, su tenencia implica que en algunas ocasiones pueden comportarse de manera irracional, en el sentido en que algunos inversionistas ven oportunidades en el mercado inmobiliario y tienden a adquirir el bien presionando la demanda y, en consecuencia, el precio del mercado. Esta situación puede ilustrarse con los casos de España y Estados Unidos, donde las facilidades que otorgaron las entidades crediticias desencadenaron una onda 
especulativa en torno a la tenencia del inmueble. Por lo tanto, hay dos razones que explican la formación de una posible burbuja inmobiliaria: la especulación y la expansión crediticia.

La burbuja inmobiliaria arranca en el seno del propio mercado inmobiliario, por razones de índole no solo económica, también está realimentada, sobre todo en una segunda etapa, por abundante crédito hipotecario favorecido por políticas monetarias expansivas y bajas tasas de interés. Durante la expansión de la burbuja, la oferta de bienes no crece en función de las necesidades reales de la sociedad sino en pro de satisfacer a los especuladores y rentistas; es así como los bienes construidos son más de aquellos que la sociedad puede consumir y pagar, y cuando esto sucede toda la estructura especulativa se viene abajo.

Para poder determinar el precio de la vivienda, se debe conocer cuáles son los determinantes del precio de la misma, que pueden estar relacionados con factores macroeconómicos, demográficos y financieros (Sánchez, 2013). Factores tales como el precio de la tierra, el nivel de empleo, el costo de mantenimiento de la vivienda, los impuestos a la posesión de vivienda, los incentivos tributarios, el incremento de la renta, las expectativas de cambio en los precios y el incremento de la inversión extranjera, pueden estar relacionados con variaciones del precio de los inmuebles (Poterba, 1984).

Poterba (1984), propone un modelo para determinar el precio teórico de la vivienda a partir de los factores determinantes anteriormente descritos, los cuales se resumen en la siguiente fórmula:

Siendo:

$$
R(H)=\left(r+\delta+m+\rho+\pi^{e}\right) p
$$

$R(H)$ : el alquiler real o renta imputada

$\mathrm{r}$ : la tasa de interés del préstamo hipotecario

$\delta$ : la tasa de depreciación

$m$ : el costo de mantenimiento

$\rho$ : la prima de riesgo

$\pi^{e}$ : la tasa de revalorización esperada del capital

$p$ : el precio real de la vivienda 
El modelo implica la condición de ausencia de arbitraje, es decir, que el costo de tener una vivienda en propiedad debe ser igual a la renta que se obtiene de esta. Este concepto teórico del precio es fundamental para identificar los principales causantes de los ciclos inmobiliarios, donde la tasa de interés de los créditos hipotecarios incide de manera significativa en el alza en los precios y es uno de los factores más determinantes para la explicación de los ciclos.

De acuerdo a Poterba (1984), la vivienda genera una renta la cual establece una función inversa con los servicios que demanda la tenencia de la misma, los cuales son consumidos hasta un valor equivalente a sus costos. En situación de equilibrio, los costos marginales deben ser igual a los beneficios marginales o renta marginal $R(H)$ por unidad de vivienda (H Stock). La vivienda tiene un precio real $p$ y a su vez el inmueble genera unos costos, como la prima de riesgo $\rho$, el costo de mantenimiento $m$, la depreciación $\delta$, y la tasa de interés de la hipoteca $r$. Por lo tanto $R=R(h(H)), R^{\prime}<0$, donde la renta real es el inverso de la función de los servicios de la vivienda.

De otra parte, establece que el cambio en el precio real del stock de la vivienda en una situación de un equilibrio del mercado de activos está dado como: $\dot{p}=-R(H)+v p$; es decir, una variación de los precios de la vivienda es igual a la relación inversa entre los servicios de la vivienda $v p$ y la renta real de la vivienda $R(H)$. Y define Poterba (1984):

$$
\nu=\delta+m+(1-\theta)(r-\mu)-\pi
$$

Dado el stock inicial de la vivienda $H$ y el precio real $p$.

$v$ : representa los costos de los servicios de la vivienda

Donde $(1-\theta)$ es la tasa marginal del impuesto sobre la renta o deducción de impuestos sobre la propiedad.

$\mu$ : tasa de impuestos por propiedad o pasivo fiscal

$r$ : tasa de interés real

$\pi_{H}$ : inflación del stock de la vivienda

Por lo tanto, el precio nominal de la vivienda es igual a la suma de la inflación general $\pi$ y la inflación del precio real de la vivienda $\pi_{\rho}$, entonces: 


$$
\pi_{\rho}=\frac{\dot{p}}{p}=\pi_{H}-\pi
$$

Se puede establecer que el precio nominal de la vivienda está dado por la variación de los precios en el tiempo y la diferencia con la tasa de inflación. Un aumento en los precios de la vivienda genera un aumento en la tasa de inflación del stock general de la vivienda.

\section{Aspectos metodológicos del análisis de la presencia de burbujas inmobiliarias}

Desde el punto de vista metodológico y para el caso del análisis de la presencia de una posible burbuja inmobiliaria ${ }^{1}$, a nivel internacional algunos países han presenciado procesos especulativos en el mercado de vivienda que han dado lugar al incremento desmesurado de los precios, conllevando a la aparición de burbujas que han generado crisis de confianza afectando negativamente las decisiones de los inversionistas de los demás sectores económicos, desencadenando crisis financieras (Idrovo, 2013).

Con el fin de determinar si hay o no presencia de burbujas, se han desarrollado algunas metodologías basadas en el seguimiento y análisis de los índices de precios de la vivienda, para determinar si el comportamiento de la actividad se debe a conductas especulativas o a un aumento de la demanda inmobiliaria. Una metodología se basa en el análisis de cointegración concebida como un indicador de precios y sus variables fundamentales. Mediante una prueba de cointegración, se puede descartar la presencia de una burbuja inmobiliaria, a través del análisis de una serie no estacionaria. Previamente es importante efectuar una prueba de raíz unitaria para preseleccionar estadísticamente

1 El término burbuja inmobiliaria se refiere al aumento rápido y continuo de los precios de los inmuebles, provocados por la presencia de expectativas ante incrementos adicionales y el aumento de los niveles de ganancia, atractivo para los inversionistas (Cediel, 2015, p. 234). 
las series que harán parte del análisis de cointegración. Las pruebas de raíz unitaria de Dickey y Fuller en sus múltiples versiones hacen parte de las técnicas de series de tiempo univariadas, y su objetivo es determinar si una serie de tiempo muestra un comportamiento explosivo (Cediel et al, 2015, p. 250). El objetivo de esta metodología es identificar las variables macroeconómicas que inciden en la estabilidad de los precios de la vivienda a largo plazo y garantizan su equilibrio.

Otra metodología utilizada para determinar burbujas en el precio de los activos es la prueba de signo, propuesta por So y Shin en 2001, la cual se utiliza para detectar caminatas aleatorias versus procesos alternativos generales y no estacionarios. Según Fernández y Uribe, la prueba de signo es consistente ante una amplia gama de procesos no lineales, estacionarios o explosivos. Adicionalmente, no varía ante transformaciones monotónicas de datos.

En la literatura sobre burbujas, principalmente se encuentran pruebas econométricas que permiten determinar si los comportamientos de las series de tiempo de los precios de las viviendas presentan procesos estocásticos o tienen momentos explosivos que se pueden determinar mediante las pruebas anteriormente mencionadas. Sin embargo, existen en la literatura otro tipo de modelos para determinar burbujas como el presentado por Abad en 2013, que se basó en una modificación del modelo Poterba para determinar el precio teórico de la vivienda y lo comparó con el valor de la vivienda observado, encontrando cambios significativos en los precios y así estableciendo una posible burbuja del precio.

Para contrastar los resultados obtenidos, Abad acude a la literatura propuesta por Froot y Obtsfeld, quienes definen un tipo de burbuja llamada "burbuja intrínseca", la cual según los autores, no obedece a factores exógenos ajenos a las variables, sino a una sobrerreacción de los agentes a la variación de los fundamentales que determinan el precio de los activos.

El modelo se basa en el cálculo del precio de las acciones, el cual depende del valor presente de los beneficios futuros, más un término de burbuja racional, siendo este último, una función no lineal de los dividendos que paga la acción, por lo cual depende exclusivamente de 
los fundamentales que determinan el precio de la acción y la percepción de los agentes sobre su impacto en el precio.

Teniendo en cuenta que el modelo es utilizado para la valoración de acciones, para la aplicación al mercado de vivienda el valor de los dividendos es reemplazado por el valor de la renta fundamental del modelo Poterba y los factores fundamentales determinarían el precio teórico.

Con esta variación del modelo, los resultados obtenidos permitieron determinar que efectivamente el precio de la vivienda se debía a la sobrerreacción de los agentes a los fundamentales (Abad Sánchez, 2013).

\section{Conclusiones}

La vivienda es uno de los principales activos que representa riqueza para muchos hogares y genera estabilidad y confianza, en el objetivo social de lograr una mejor calidad de vida de la población. Su análisis implica indagar sobre la forma como los hogares adquieren el inmueble, ya sea con recursos propios o financiado con alguna entidad bancaria. La dificultad que afrontan las personas es la disponibilidad de ingresos que garanticen su adquisición y pago, este flujo depende esencialmente de la forma en que se adquiere, bien sea a través del trabajo, la rentabilidad en un portafolio de inversión, la riqueza, los alquileres, las tasas de interés por captaciones o las herencias, sin embargo, su distribución generalmente es desigual.

A través del análisis teórico se puede afirmar que existe una estrecha relación entre la riqueza, la vivienda y el ingreso, pero esta equivalencia está afectada por la manera como se ha distribuido el ingreso social. El ingreso como flujo, es la variable que permite adquirir el inmueble y satisfacer una necesidad importante de los hogares como es el acceso a la vivienda, lo cual garantiza una mejor calidad de vida, pero la dificultad para muchas familias ha sido mantener la generación durante el ciclo de vida laboral, dado que en muchas situaciones lograr este bien requiere financiación a largo plazo, por lo tanto el problema es que coincida el ciclo de vida laboral de la persona, con el plazo de pago de las cuotas hipotecarias que garanticen la propiedad del inmueble. 
También es importante considerar que en el mercado de vivienda, los precios juegan un papel relevante, dado que determinan la construcción de viviendas nuevas para los hogares. Pero como los mercados afrontan fallas y se observan situaciones en donde la demanda supera la oferta, se presentan circunstancias donde el aumento de precios genera nuevas oportunidades para muchos inversionistas del sector de la construcción, que se relacionan estrechamente con la presencia de fenómenos especulativos que afectan el mercado inmobiliario y ocasionan oscilaciones irregulares en los precios. Esta relación entre precios, demanda, escasez de tierras, especulación y confianza, ha conllevado a la generación de crisis financieras estrechamente relacionadas con las crisis hipotecarias, que amenazan con la formación de posibles burbujas especulativas de precios, que en el mediano plazo afectan la estabilidad de los mercados y el bienestar de la población.

De otra parte, la financiación de la vivienda ha estado estrechamente relacionada con la política monetaria, pues las tasas de interés como mecanismo de financiación han jugado un papel determinante en el otorgamiento de los créditos hipotecarios. Se ha establecido una especie de mecanismo de transmisión de la política monetaria que incide en el costo de uso de la vivienda, las expectativas, la riqueza y los precios de los inmuebles. Si el Gobierno decide incentivar la demanda, baja la tasa de interés del mercado para estimular la inversión y por ende la compra de nuevas unidades, entonces, este mecanismo de transmisión se asocia con un aumento de la oferta monetaria; sin embargo, en el mediano plazo un aumento en la demanda dados estos incentivos, puede generar fenómenos especulativos que inciden en el aumento de los precios de la finca raíz, hasta el punto de que pueden encarecer la adquisición y a nivel macroeconómico ocasionar fenómenos inflacionarios, que afectan el consumo y el ahorro de los tenedores de obligaciones hipotecarias.

Desde diferentes perspectivas teóricas que explican el problema de la distribución de la riqueza, y sus formas de generación han sido tema de análisis en los procesos de acumulación, (pues en una economía de mercado donde la propiedad privada es la manera como las personas pueden acceder a mejores condiciones de vida), esto no 
garantiza que muchos hogares cuenten con las mismas condiciones de acceso a estos recursos. Por ello, los créditos han jugado un papel determinante para adquirir vivienda propia, pero en una sociedad que afronta problemas de desempleo o muestra elevados índices de informalidad, las exigencias de las formas de financiación limitan el acceso a estos bienes, entonces se puede establecer una estrecha relación entre la exclusión financiera, la desigualdad y las realizaciones. El manejo de la política monetaria debe ir de la mano con los programas que el Gobierno diseña para que muchos sectores excluidos de la población accedan a la tenencia de estos activos y mejoren su situación de bienestar. Sin embargo, el Gobierno debe garantizar una política de manejo integral que sea coherente con una política de generación de empleos formales y estables y una mayor equidad en la distribución de la renta social.

Los modelos que pretenden determinar la existencia de burbujas, buscan comprobar si hay momentos en que los precios de las viviendas pueden estar sobrevalorados frente a los valores teóricos de las mismas. Los resultados son variados y no existe un consenso que permita definir una metodología única para el cálculo de las burbujas, por lo tanto, la determinación de una burbuja inmobiliaria en los países en estudio, se deberá realizar teniendo presente la existencia de los datos en los países de análisis, la calidad de los mismos y la pertinencia con referencia no solo al precio en que se negocian en el mercado inmobiliario los activos, sino las posibles causas que puedan contribuir a la exacerbación de los precios de la vivienda.

\section{Referencias}

Abad Sánchez, J. (2013). Precios de la vivienda, sobrevaloración y burbuja. Madrid, España: Universidad Complutense de Madrid.

Alonso, M. A., Bagus, P., \& Rallo, J. R. (2011). La crisis subprime a la luz de la teoría austriaca del ciclo económico: expansión crediticia, errores de decisión y riesgo moral/The Subprime Crisis in the Light of the Austrian Business Cycle Theory: Credit Expansion, Decision Errors and Moral H. Revista de Economía Mundial, 28. 
Arriaga, N. R. (2012). Ciclos inmobiliarios y precios de la vivienda: España, EUA y Reino Unido. (U. A. Metropolitana, Ed.) Análisis Económico, XXVII (66), 2-253.

Banco Central de Chile. (2014, junio). Indice de precios de vivienda en Chile. (B. C. Chile, Ed.). Estudios Económicos y Estadísticos(107), 1-30.

BBVA. (2014). Observatorio Económico Latam. BBVA, Bogotá D. C., Colombia.

Cediel, S. V. (2015, primer semestre). ¿Hay una burbuja inmobiliaria en Bogotá? Un estudio por segmentos de mercado. (U. Nacional, Ed.) Revista de Economía Institucional, 233-257.

Cerezo, A. S. (2014, enero-junio). Boom en el sector inmobiliario en Bolivia. Burbuja o fundamentos económicos. Revista de Análisis, 20, 75-118.

COFACE. (2015, diciembre). http://www.coface.es/Noticias-y-Publicaciones. Recuperado de://C:/Users/SecAdmisiones/Downloads/151210_Construcci\%C3\%B3n+en+Francia.pdf

Hayek, F. (2008). Prices, productions and other works the bussines cycle and the gold standard. (J. T. Salerno, Ed.) Auburn, AL.

Idrovo, B. Y. (2013). Una aplicación de métodos de detección de burbuja inmobiliaria: caso Chile. MPRA Paper N 44741, posted 5. March 2013 11:51 UTC, Cámara Chilena de la Construcción, Gerencia de Estudios, Santiago de: Chile, Chile.

López, E. A. (2009). El efecto riqueza de la vivienda en Colombia. Bogotá D. C., Colombia: Banco de la República.

Marx, K. (2008, octubre). http://aristobulo.psuv.org.ve/wp-content/ uploads/2008/10/marx-karl-el-capital-tomo-i1.pdf, Archivo digital de Fidel Ernesto Vásquez. Recuperado el 20 de julio de 2016, de http:// aristobulo.psuv.org.ve/wp-content/uploads/2008/10/marx-karl-el-capital-tomo-i1.pdf.

Mishkin, F. S. (2007). Housing and the monetary transmission mechanism. Cambridge, MA 02138: National Bureau of Economic Research.

Modigliani, F. (1986). Life cycle, individual thrift and the wealth of nations. (M. I. Sloan School of Management, Ed.) American Economica Review, 76(3), 297-313.

Moneo Abreu, R. (2004). Formación de ciclos en el mercado inmobiliario.

Directivos Construcción (163), 22. 
Piketty, T. (2013). El capital en el siglo XXI. (A. Goldhammer, Trad.) Cambridge, MA. Londres, Inglaterra.

Poterba, J. M. (1984, noviembre). Tax subsidies to owner-occupied housing: an asset-market approach. (T. M. Press, Ed.) The Quarterly Journal of Economics, 99(4), 729-752.

Ricardo, D. (1993). Principios de economía política y tributación. (P. Sraffa, Ed.) México D. F., México: Fondo de Cultura Económica.

Rodríguez, L. J. (2015). Una recuperación gradual de la vivienda en España. Ciudad y Territorio estudios territoriales CyTET, LVII(185), 575-588.

Sen, A. (2011). Nuevo exámen de la desigualdad. Madrid, España: Alianza Editorial.

Sen, A. (2011). ¿Igualdad de qué? Madrid, España: Editorial Alianza. Prefacio consultado en http://www.fder.edu.uy/contenido/rrll/contenido/licenciatura/documentos/sen-amartya_prefacio-e-iguadad-de-que.pdf, PDF. Recuperado el 29 de julio de 2016.

Smith, A. (1958). Investigación de la naturaleza y causa de la riqueza de las naciones. (E. Fonseca, \& F. Gabriel, Eds.) México D. F., México: Fondo de Cultura Económica.

Tobin, J. (1978). Monetary policies and the economy: the transmission mechanism. Southern Economic Journal, 421-431.

Uribe, J., \& Fernádez, J. (2016). Análisis de procesos explosivos en el precio de los activos financieros. Revista Finanzas y Economía Política, 83-103.

Von Mises, L. (1935). The theory of money and credit. Auburb, AL: Ludwing Von Mises Institute.

Von Mises, L. (2005, octubre). La teoría austriaca del ciclo económico. (I. U. ESEADE, Ed.) Revista Libertas, XII(43). Recuperado el 31 de julio de 2016, de http://www.eseade.edu.ar/files/Libertas/3_12_Mises_Teoria\%20Austriaca.pdf 
